\title{
Ocular malignant fibrous histiocytoma: clinical and histopathological characteristics
}

\author{
M MATHEW KRISHNAN,' V K KAWATRA,' C RATNAKAR,' V A RAO,' \\ AND A J VELIATH ${ }^{2}$
}

From the Departments of 'Ophthalmology and 'Pathology, Jawaharlal.Institute of Postgraduate Medical Education and Research, Pondicherry-605006, India

SUMMARY A rare case of malignant fibrous histiocytoma involving the whole eye is reported in an Oriental 60-year-old man. Six months' follow-up after exenteration did not show any evidence of local recurrence or distant metastases.

Malignant fibrous histiocytoma was first described by O'Brien and Stout ${ }^{1}$ in 1964. The orbit is a site of predilection of this tumour. ${ }^{2}$ Litricin $^{3}$ reported fibrous histiocytoma of the corneosclera in a 65-yearold woman, and Urdiales-Viedma et al. ${ }^{4}$ reported pleomorphic fibrous histiocytoma of the corneoscleral limbus in a 72-year-old woman. To our knowledge this is the first case of pleomorphic malignant fibrous histiocytoma involving the whole eye.

\section{Case report}

.A 60-year-old man was examined on 9 July 1985 in the Department of Ophthalmology, JIPMER, Pondicherry, with the complaints of gradually increasing painless swelling under the left upper lid of one year's duration and progressive loss of vision for six months. Three months later there was gradual protrusion of the same eye, with severe pain and blood stained purulent discharge.

Examination of the left eye showed swollen lids and matted cilia. On retracting the upper lid there was a nodular swelling measuring $8.5 \times 7 \mathrm{~mm}$ at the superior limbus. The whole of the bulbar conjunctiva was thickened, and the surface was irregular and firmly adherent to underlying sclera. Cornea, anterior chamber, iris, and lens were not identified. A nodular greyish mass was seen through the destroyed cornea (Fig. 1). On palpation the orbital margins were normal. The mass was tender and noncompressible. The preauricular and submandibulbar

Correspondence to M Mathew Krishnan, FICS, Department of Ophthalmology, Jawaharlal Institute of Postgraduate Medical Education and Research, Pondicherry-605006, India. lymph nodes were not enlarged. There was no perception of light. Examination of the right eye revealed immature senile cataract. The best corrected visual acuity was 6/12 (Snellen's chart). The intraocular pressure was $17.3 \mathrm{mmHg}$ (Schiøtz tonometer) and the fundus was normal. Systemic examination did not show any evidence of secondary growths.

A complete haemogram revealed no abnormality. $X$-rays of the orbits, optic foramina, and chest were normal. A clinical diagnosis of ocular tumour was made. Biopsy showed malignant tumour, but categorisation could not be made as the specimen was small. Exenteration of the left orbit was done on 28 August 1985.

\section{HISTOPATHOLOGY}

Macroscopic examination. The exenterated specimen of left orbit measured $31 \times 29.2 \times 28.7 \mathrm{~mm}$.

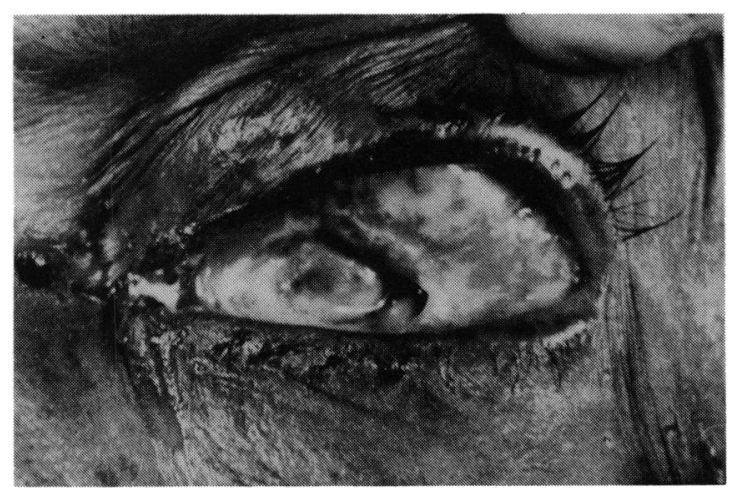

Fig. 1 Swelling at the superior limbus. Greyish mass is seen through destroyed cornea. 


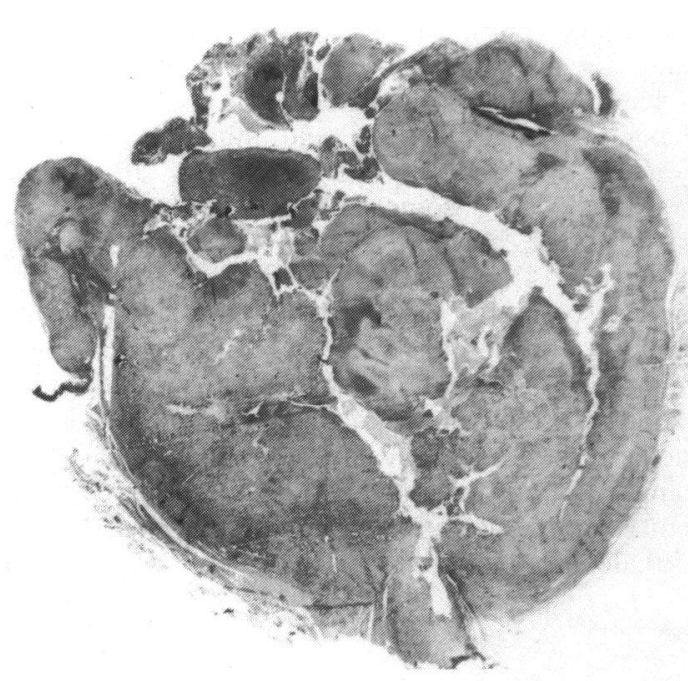

Fig. 2 Intraocular location of tumour with foci of necrosis. Tumour has come out of corneoscleral junction infiltrating the conjunctiva. Optic nerve also shows tumour infiltration. $H$ and $E$.

There was an irregular nodular elevation under the upper eyelid measuring $8.5 \times 7 \mathrm{~mm}$. The cornea was completely ulcerated and was covered by necrotic tissue. The optic nerve measured $3 \mathrm{~mm}$. Transillumination was negative in all directions. The eyeball was cut vertically. The cut section showed a lobulated greyish white tumour measuring $24.4 \times 21.0 \times 18.6$ $\mathrm{mm}$. The tumour was soft to firm in consistency, with foci of necrosis (Fig. 2). It occupied the whole eye, destroying the uveal tract and retina completely, and extended on to the optic disc. The lens was not seen. The sclera was infiltrated by the tumour just behind the equator on the inferior side and near the superior

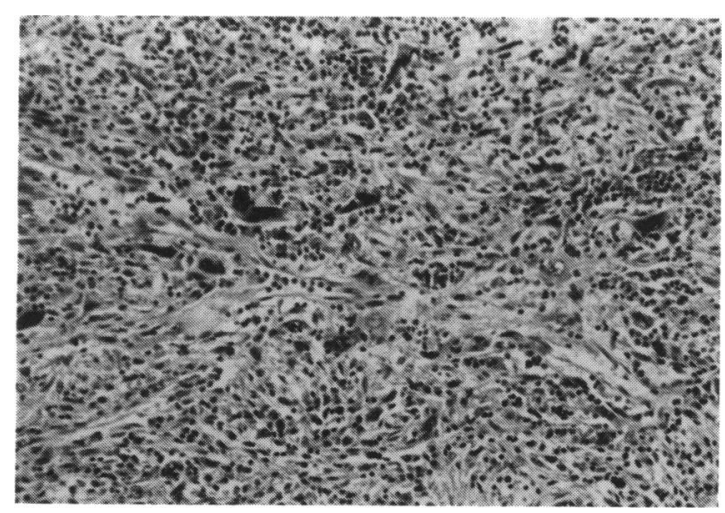

Fig. 3 Spindle shaped cells arranged in a storiform pattern. There are considerable number of giant cells and inflammatory cells. $H$ and $E$.



Fig. 4 Histiocyte-like cells. A fair number of inflammatory cells are also seen. $H$ and $E$.

limbus. The nodule under the upper lid, on cut section, was continuous with the intraocular tumour at the limbus.

Microscopic examination. The tumour showed a variable histological pattern, having a storiform pattern in some areas and pleomorphic appearance in other areas. The tumour consisted of plump spindle cells in fascicles around inconspicuous vessels (Fig. 3). These cells had abundant eosinophilic cytoplasm with a round pale-staining central nucleus. In some areas there were plump cells with pronounced atypia mixed with a considerable number of histiocytes and other inflammatory cells (Fig. 4). The giant cells had multiple deeply staining nuclei and bright eosinophilic cytoplasm (Fig. 5). Typical and atypical mitotic figures were seen. Inflammatory cells, mainly plasma cells and lymphocytes, were conspicuous in some areas. Delicate collagen fibrils separated the tumour cells.

The tumour cells on staining showed faint positivity for neutral fat, but stains for glycogen were negative. Special stains failed to reveal cross

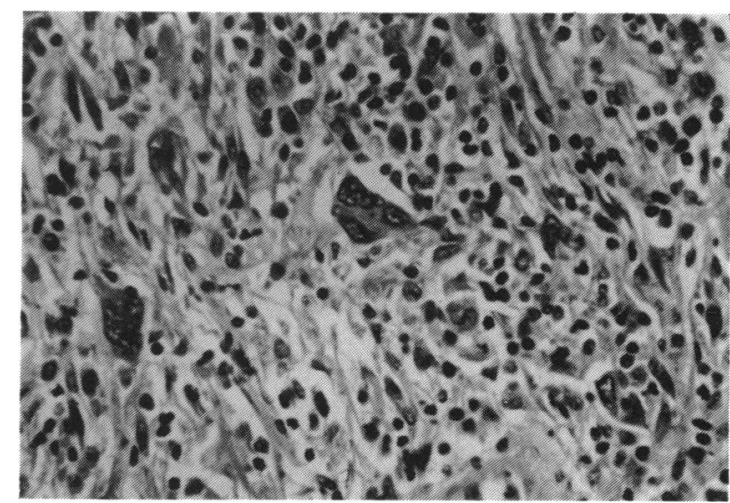

Fig. 5 Pleomorphic giant cells. $H$ and $E$. 
striations, ruling out a myogenic tumour. A diagnosis of malignant fibrous histiocytoma infiltrating the retina, uveal tract, conjunctiva, cornea, sclera, and optic disc was made.

\section{Discussion}

Malignant fibrous histiocytoma is a primitive mesenchymal tumour showing partial fibroblastic and histiocytic differentiation; it occurs commonly in late adult life. ${ }^{5}$ These tumours have a wide range of histological patterns-for example, myxoid, inflammatory, angiomatoid, with formation of bone and cartilage, pleomorphic, fibroblastic, xanthomatous, and xanthogranulomatous. ${ }^{4}$ Orientals $(2 \%)$ are less commonly affected than Caucasians $(91 \%)$ and Negroes $(7 \%){ }^{5}$ These are seldom reported because of rare occurrence in the eye. ${ }^{46}$ Males and females are equally affected with orbital tumours, ${ }^{2}$ though females are mainly subject to ocular tumours. ${ }^{3+67}$ The present case was in an Oriental 60-year-old man.

The sclera and corneal stroma are usually resistant barriers to the spread of tumour from episclera and conjunctiva. ${ }^{8}$ Litricin $^{3}$ reported a case of fibrous histiocytoma of the corneosclera which invaded the iris, possibly following a perforation at the limbus during sclerokeratoplasty 11 months prior to enucleation. Delgado-Partida et al. ${ }^{6}$ reported a case of malignant fibroxanthoma in which the ciliary body was possibly invaded secondarily from the conjunctiva. In our case it is difficult to pinpoint the site of origin of the tumour. However, as the tumour is mainly intraocular in location, it seems more plausible to think that this tumour arose from retina and, after destroying the sclera and other structures, went out at the superior limbus and presented as a nodule under the upper lid.

The prognosis of malignant fibrous histiocytoma depends on depth, size, and inflammatory component. ${ }^{5}$ Recurrences are frequent after local excisions. $^{36}$ It is sometimes quite difficult on the basis of the histopathology to predict biological behaviour. ${ }^{2}$ Death from local extension and distant metastases has been reported in malignant orbital tumours. ${ }^{2}$ In the present case the history was of one year's duration and follow-up of six months after exenteration without local recurrence or distant metastases. The clinical course so far has been benign, though histologically the tumour is malignant. The same condition has been observed by Urdiales-Viedma et al. ${ }^{4}$

\section{References}

1 O'Brien JE, Stout AP. Malignant fibrous xanthomas. Cancer 1964; 17: 1445-58.

2 Font RL. In: Jones IS, Jakobiec FA, eds. Diseases of the orbit (1). Hagerstown: Harper and Row, 1979: 466-8.

3 Litricin O. Fibrous histiocytoma of the corneosclera. Arch Ophthalmol 1983; 101: 426-8.

4 Urdiales-Viedma $M$, Moreno-Prieto $M$, Martos-Padilla $S$. Pleomorphic fibrous histiocytoma of the corneoscleral limbus. Am J Ophthalmol 1983; 95: 560-1.

5 Weiss SW, Enzinger FM. Malignant fibrous histiocytoma. Cancer $1978 ; 41$ : 2250-66.

6 Delgado-Partida P, Rodriguez-Trujillo F. Fibrosarcoma (malignant fibroxanthoma) involving conjunctiva and ciliary body. Am J Ophthalmol 1972; 74: 479-85.

7 Jakobiec FA. Fibrous histiocytoma of the corneoscleral limbus. Am J Ophthalmol 1974; 78: 700-6.

8 Irvine AR. Epibulbar squamous cell carcinoma and related lesions. Int Ophthalmol Clin 1972; 12 : 71.

Accepted for publication 31 October 1986. 